\title{
Genus-One Surface Registration via Teichmüller Extremal Mapping
}

\author{
Ka Chun Lam $^{1}$, Xianfeng Gu${ }^{2}$, Lok Ming Lui ${ }^{1}$ \\ 1 Department of Mathematics, The Chinese University of Hong Kong \\ 2 Department of Computer Science, State University of New York at Stony Brook \\ $\{\mathrm{kclam}, 1 \mathrm{mlui}\} @_{\mathrm{math}}$. cuhk. edu.hk, gu@cs.sunysb. edu
}

\begin{abstract}
This paper presents a novel algorithm to obtain landmark-based genus-1 surface registration via a special class of quasi-conformal maps called the Teichmüller maps. Registering shapes with important features is an important process in medical imaging. However, it is challenging to obtain a unique and bijective genus-1 surface matching that satisfies the prescribed landmark constraints. In addition, as suggested by [11], conformal transformation provides the most natural way to describe the deformation or growth of anatomical structures. This motivates us to look for the unique mapping between genus- 1 surfaces that matches the features while minimizing the maximal conformality distortion. Existence and uniqueness of such optimal diffeomorphism is theoretically guaranteed and is called the Teichmüller extremal mapping. In this work, we propose an iterative algorithm, called the Quasi-conformal (QC) iteration, to find the Teichmüller extremal mapping between the covering spaces of genus-1 surfaces. By representing the set of diffeomorphisms using Beltrami coefficients (BCs), we look for an optimal $\mathrm{BC}$ which corresponds to our desired diffeomorphism that matches prescribed features and satisfies the periodic boundary condition on the covering space. Numerical experiments show that our proposed algorithm is efficient and stable for registering genus- 1 surfaces even with large amount of landmarks. We have also applied the algorithm on registering vertebral bones with prescribed feature curves, which demonstrates the usefulness of the proposed algorithm.
\end{abstract}

\section{Introduction}

Surface registration is increasingly used in morphometric analysis. By finding a meaningful one-to-one correspondence between anatomical surfaces, statistical shape analysis, processing of signals on anatomical surfaces (e.g., denoising or filtering) and age-related comparison can be achieved. In landmark-based registrations, landmarks are extracted to guide the registration process to obtain a meaningful transformation. Through labeling landmarks, medical experts and doctors can get involved in the process to assure good correspondences between the surfaces. However, obtaining a unique and bijective registration that matches features consistently is generally challenging, especially when a large number of landmark constraints are enforced. Developing an effective algorithm for registration is therefore very important.

Surface registration between simple surfaces, such as simply-connected open surfaces or genus-0 closed surfaces, has been extensively studied. However, as far as we

P. Golland et al. (Eds.): MICCAI 2014, Part III, LNCS 8675, pp. 25-32, 2014.

(C) Springer International Publishing Switzerland 2014 
know, very few literatures have been reported on the registration between genus-one surfaces. The high-genus topology of the surfaces poses a great challenge to register the surfaces. For example, the vertebral shape is commonly analyzed through simple geometric measurements of dimensions, which only describe limited features of the complex vertebral shape. In order to provide a more comprehensive description, a more sophisticated landmark-based surface registration is essential for analyzing both local and global geometric information of a vertebral shape.

Motivated by this, we are interested in searching for the unique and bijective landmark-matching diffeomorphism which minimizes the maximal conformality distortion. The conformality distortion measures how far the mapping is deviated from a conformal mapping, and hence it measures the local geometric distortion.. The existence and uniqueness of such a mapping is theoretically guaranteed by the Quasi-conformal Teichmüller theory [2], and is named the Teichmüller extremal map. In this paper, we propose a novel algorithm to compute the Teichmüller extremal map between genus-1 surfaces. Experiments on vertebral bones are also reported to show the accuracy and effectiveness of the proposed algorithm.

\section{Previous Work}

Landmark-based registration has been widely studied in medical imaging, computer graphics and computer visions. Various algorithms have been proposed to match feature landmarks consistently. For example, Bookstein et al.[1] proposed to obtain a registration that matches landmarks as much as possible using a thin-plate spline regularization (or biharmonic regularization). Gu et al. [45] proposed to compute the conformal parameterizations of human brain surfaces for registration using harmonic energy minimization and holomorphic 1 -forms. Conformal registration is advantageous for the preservation of the local geometry. However, it cannot align landmark features, such as sulci landmarks on brain surfaces, consistently. Sometimes, deformation between objects might not be conformal. Instead, certain amount of angular distortion could be introduced. To tackle with this problem, quasi-conformal mappings have been applied to obtain surface registration with bounded conformality distortion [8 9]. Introduction of time-dependent vector fields for registration is also proposed [73]. For example, Glaunés et al. in [3] presented to generate large deformation diffeomorphisms of a sphere, with given displacements of a finite set of template landmarks. The time dependent vector fields facilitate the optimization procedure, but the computational cost of the algorithm is comparatively more expensive.

\section{Mathematical Background}

\subsection{Quasi-Conformal Map}

Quasi-conformal maps are orientation preserving homeomorphisms between Riemann surfaces with bounded conformality distortion. Intuitively, they take infinitesimal 
circles to infinitesimal ellipses of bounded eccentricity. Mathematically, $f: \mathbb{C} \rightarrow \mathbb{C}$ is quasi-conformal provided that it satisfies the Beltrami equation:

$$
\frac{\partial f}{\partial \bar{z}}=\mu(z) \frac{\partial f}{\partial z}
$$

for some complex-valued function $\mu$ satisfying $\|\mu\|_{\infty}<1$. The function $\mu$ is a measure of non-conformality and is named the Beltrami coefficient. In particular, a map $f$ is conformal at $p$ if $\mu(p)=0$. Denote $i=\sqrt{-1}$ and $f=u+i v$. From the Beltrami equation (1),

$$
\mu(f)=\frac{\left(u_{x}-v_{y}\right)+i\left(v_{x}+u_{y}\right)}{\left(u_{x}+v_{y}\right)+i\left(v_{x}-u_{y}\right)}
$$

Let $\mu(f)=\rho+i \tau$. We have the following linear combinations between $u_{x}, u_{y}, v_{x}$ and $v_{y}$ :

$$
\left\{\begin{array}{r}
-v_{y}=\alpha_{1} u_{x}+\alpha_{2} u_{y} \\
v_{x}=\alpha_{2} u_{x}+\alpha_{3} u_{y}
\end{array} ; \quad\left\{\begin{array}{r}
-u_{y}=\alpha_{1} v_{x}+\alpha_{2} v_{y} \\
u_{x}=\alpha_{2} v_{x}+\alpha_{3} v_{y}
\end{array}\right.\right.
$$

where $\alpha_{1}=\frac{(\rho-1)^{2}+r^{2}}{1-\rho^{2}-r^{2}} ; \alpha_{2}=\frac{2 r}{1-\rho^{2}-r^{2}} ; \alpha_{3}=\frac{1+2 \rho+\rho^{2}+r^{2}}{1-\rho^{2}-r^{2}}$. By taking divergence on both sides of equations (3), we obtain

$$
\nabla \cdot\left(A\left(\begin{array}{l}
u_{x} \\
u_{y}
\end{array}\right)\right)=0 ; \quad \nabla \cdot\left(A\left(\begin{array}{l}
v_{x} \\
v_{y}
\end{array}\right)\right)=0, \quad \text { where } A=\left(\begin{array}{ll}
\alpha_{1} & \alpha_{2} \\
\alpha_{2} & \alpha_{3}
\end{array}\right)
$$

According to the Quasi-conformal Teichmüller theory, a quasi-conformal map can be uniquely determined up to Mobiüs transformations. Ambiguity of the Mobius transformation can be eliminated by providing three points correspondence, in which a unique solution can be obtain from equation (4).

\subsection{Teichmüller Extremal Map}

Let $\mu(f)$ be the Beltrami coefficient of $f$. Define the maximal dilation of $f$ to be:

$$
K(f)=\frac{1+\|\mu(f)\|_{\infty}}{1-\|\mu(f)\|_{\infty}} .
$$

Using maximal dilation, we can define extremal map as:

Definition 1 Let $f: S_{1} \rightarrow S_{2}$ be a quasi-conformal mapping between $S_{1}$ and $S_{2} . f$ is said to be an extremal mapping if for any quasi-conformal mapping $h: S_{1} \rightarrow S_{2}$ isotopic to $f$ relative to the boundary,

$$
K(f) \leq K(h)
$$

It is uniquely extremal if the inequality (6) is strict when $h \neq f$.

Another kind of mapping, called the Teichmüller mapping, is closely related to the extremal mapping. Teichmüller mapping is defined as follows: 
Definition 2 Let $f: S_{1} \rightarrow S_{2}$ be a quasi-conformal mapping. $f$ is said to be a Teichmüller mapping associated to the quadratic differential $q=\varphi d z^{2}$ where $\varphi: S_{1} \rightarrow$ $\mathbb{C}$ is a holomorphic function if its associated Beltrami differential is of the form:

$$
\mu(f)=k \frac{\bar{\varphi}}{|\varphi|}
$$

for some constant $k<1$ and quadratic differential $q \neq 0$ with $\|q\|_{1}=\int_{S_{1}}|\varphi|<\infty$.

Let $S_{1}$ and $S_{2}$ be Riemann surfaces with the same topology. Let $\left\{p_{i}\right\}_{i=1}^{n} \in S_{1}$ and $\left\{q_{i}\right\}_{i=1}^{n} \in S_{2}$ be the corresponding interior landmark constraints. A Teichmüller mapping $f$ between $S_{1}$ and $S_{2}$, which satisfies the landmark constraints, is actually the unique extremal map. With this, both uniqueness and existence of landmark matching Teichmüller extremal map can be guaranteed. We can therefore obtain a unique landmark matching registration by searching for an optimal Beltrami coefficient whose maximal dilatation is the minimum. For details, please refer to [2].

\section{Proposed Algorithms}

In this section, we explain our algorithm for obtaining a feature aligned Teichmüller extremal mapping between genus- 1 surfaces. The basic idea is to first embed the surfaces into their universal covering spaces and find the Teichmüller extremal mapping between their conformal parameterizations.

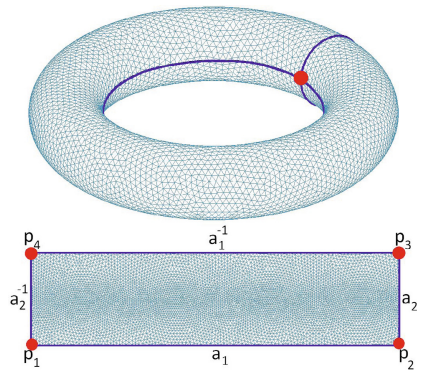

Fig. 1. Torus \& $\Omega$

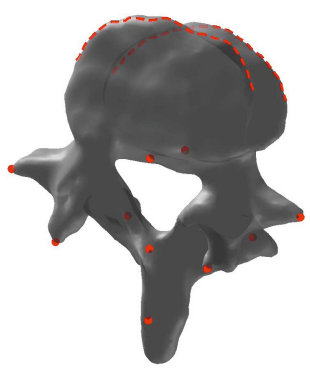

Fig. 2. Vertebral bone 1

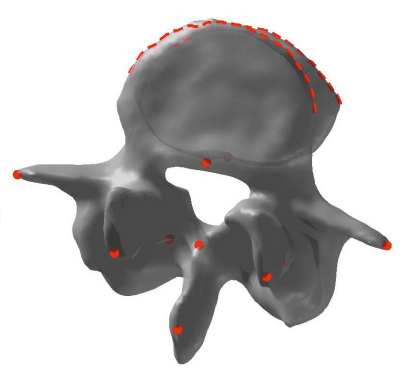

Fig. 3. Vertebral bone 2

\subsection{Embed Genus-One Surface into the Euclidean Plane}

The embedding of the genus- 1 surface is computed using the Ricci flow method introduced by Gu et al. [6]. The basic idea of Ricci flow is to conformally deform the metric $g=\left(g_{i j}(t)\right)$ according to its induced Gaussian curvature $K(t)$. Mathematically, we have

$$
\frac{d g_{i j}(t)}{d t}=-2(K(t)-\bar{K}) g_{i j}(t)
$$

where we set $\bar{K}=0$ for genus-one to be the target curvature. Convergence of this process is guaranteed by Hamilton's theorem. $g(\infty)$ is the desired uniformization metric. 
Let $S$ be a genus-1 surface and $p$ be a base point for $S$. Two closed loops based at $p$ are introduced to slice the genus- 1 surface into the fundamental domain. With the uniformization metric, the fundamental domain can be conformally embedded onto a $2 \mathrm{D}$ domain $\Omega \in \mathbb{R}^{2}$, called the fundamental polygon (See Figure 1). Denote the boundaries and vertices of the polygon as $\left\{a_{1}, a_{2}, a_{1}^{-1}, a_{2}^{-1}\right\}$ and $\left\{p_{i}\right\}$ respectively. The boundary pairs $\left\{a_{1}, a_{1}^{-1}\right\},\left\{a_{2}, a_{2}^{-1}\right\}$ and vertices $\left\{p_{i}\right\}$ correspond to the closed loop and the single based point introduced. Note that $a_{i}$ and $a_{i}^{-1}, i=1,2$ are related by $\varphi_{i}\left(a_{i}\right)=a_{i}^{-1}$, where $\varphi_{i}$ are translations in $\mathbb{R}^{2}$. Therefore, periodic constraints are enforced in the boundaries of the fundamental polygon. With this conformal parameterization, registration can be done on the fundamental domains instead of the complex genus- 1 surfaces. For details, please refer to [6]10].

\subsection{Computing the Teichmüller Extremal Mapping between Parameter Domains}

Let $\Omega_{1}$ and $\Omega_{2}$ be the fundamental polygons of two genus-1 surfaces $S_{1}$ and $S_{2}$ respectively. Denote the boundaries and vertices of $\Omega_{1}$ and $\Omega_{2}$ by $\left\{a_{1}, a_{2}, a_{1}^{-1}, a_{2}^{-1}\right\}$, $\left\{p_{i}^{S_{1}}\right\}$ and $\left\{b_{1}, b_{2}, b_{1}^{-1}, b_{2}^{-1}\right\},\left\{p_{i}^{S_{2}}\right\}$ respectively. As the boundary cuts of $S_{1}$ and $S_{2}$ may not be consistent, only periodic constraints are considered during the registration. Let $\left\{r_{k}\right\}_{k=1}^{n}$ and $\left\{q_{k}\right\}_{k=1}^{n}$ be the landmark correspondences on $\Omega_{1}$ and $\Omega_{2}$ respectively. Mathematically, the problem of finding Teichmüller extremal mapping between the fundamental domains can be formulated as follows:

$$
f=\operatorname{argmin}_{f: \Omega_{1} \rightarrow \Omega_{2}}\|\mu(f)\|_{\infty}
$$

subject to:

- $\mu(f)=k \frac{\bar{\varphi}}{|\varphi|}$ where $0 \leq k<1$ and $\varphi: \Omega_{1} \rightarrow \mathbb{C}$ is integrable holomorphic;

- $\varphi_{i}\left(f\left(a_{i}\right)\right)=f\left(a_{i}^{-1}\right)$ for $i=1,2$; (Periodic constraints)

- $f\left(p_{i}^{S_{1}}\right)=p_{i}^{S_{2}}$ for $i=1, \ldots, 4$; (Base points consistency)

- $f\left(r_{k}\right)=q_{k}$ for $k=1,2, \ldots, n$. (Landmark constraints)

To solve the above minimization problem, we propose an iterative scheme called the Quasi-conformal (QC) iteration. The basic idea is to find a path in the space of all Beltrami coefficients, which approaches from $\mu=0$ to the unique admissible Beltrami coefficient $\nu^{*}$ of Teichmüller type. The process is summarized in Algorithm 1. For the convergence of Algorithm 1, please refer to [12].

\section{Experimental Results}

To evaluate the proposed algorithm, we apply it on the vertebral bones to compute the Teichmüller extremal map between 5 pairs of vertebral bones with prescribed feature points and landmark curves as landmarks (See Figure 2 \& 3). There are two landmark curves labeled on the top and bottom side of the cortical rim and ten features marked on other parts of each vertebral bone. To register between a pair of vertebral bones, we first parameterize them into the fundamental domains $\Omega_{1}$ and $\Omega_{2}$ by the Ricci flow 


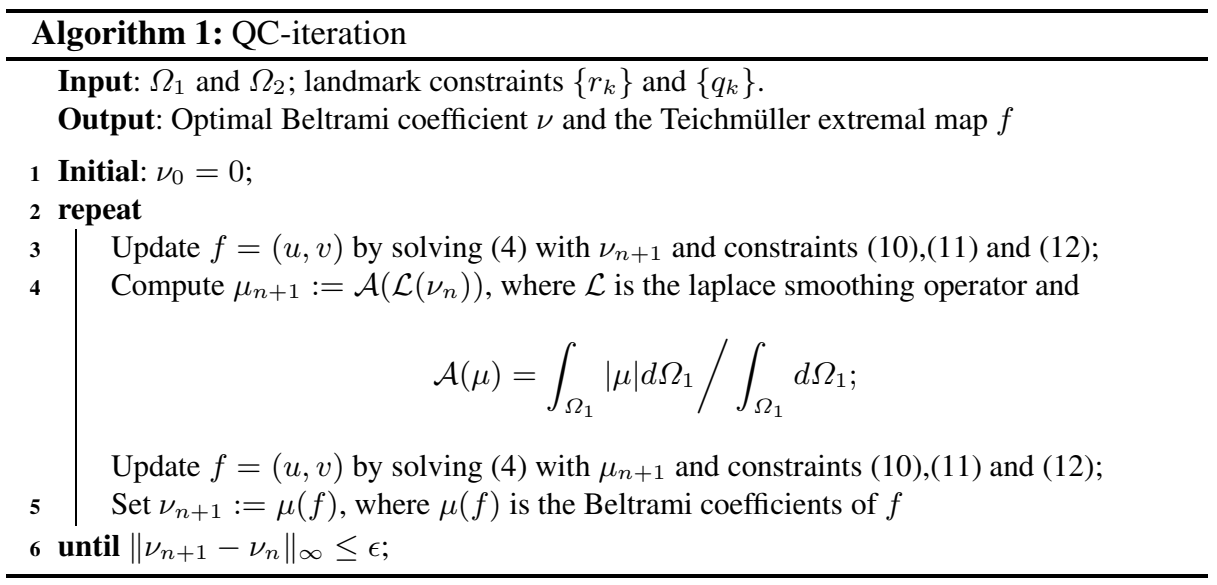

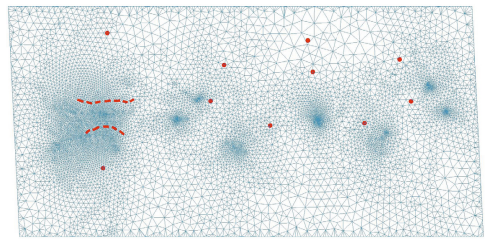

Fig. 4. Fundamental polygon $\Omega_{1}$


Fig. 6. Vertebral bone $S_{1}$ Fig. 7. Resultant registration



Fig. 5. Registered polygon and $\Omega_{2}$



Fig. 8. $|\mu|$ on surface

method. Using the QC iteration, the Teichmüller extremal mapping $f: \Omega_{1} \rightarrow \Omega_{2}$ which satisfies the landmark constraints is obtained. Since no hard constraints is enforced on the cutting boundaries in the algorithm, the cutting boundaries of $\Omega_{1}$ can move freely on the universal covering space, which satisfy the periodic conditions. Figure 5 shows the obtained Teichmüller extremal map between the covering spaces. Once the Teichmüller extremal map is computed, we can obtain the registration between the vertebral bones $S_{1}$ and $S_{2}$ by a composition of functions $\phi_{2}^{-1} \circ f \circ \phi_{1}=T: S_{1} \rightarrow S_{2}$. The resultant registration is shown in Figure 7 The mesh is obtained by deforming the source vertebral bone (Figure 6) to the target surface (Figure 3). Landmark curves and 
Table 1. Summary of the comparison experiment

\begin{tabular}{c||cccccc}
\hline Method & $e_{\max }$ & $e_{\operatorname{mean}}$ & $\|\mu\|_{\infty}$ & $S D(|\mu|)$ & $d_{H}$ & Time $(\mathrm{s})$ \\
\hline Proposed & 0 & 0 & 0.4193 & 0.0147 & 0.82 & $10.91 \mathrm{~s}$ \\
rigid ICP & 0.1467 & 0.0389 & $1.08 e^{-13}$ & $5.33 e^{-15}$ & 12.44 & $4.46 \mathrm{~s}$ \\
non-rigid ICP & 0.0798 & 0.0402 & 0.9841 & 0.1710 & 4.55 & $223.07 \mathrm{~s}$ \\
\hline
\end{tabular}

feature points are exactly matched after the registration process. Figure 8 also shows $|\mu(f)|$ of the Teichmüller Extremal mapping, which is represented by the color on the vertebral bone surface. An even color distribution on the surface and a small standard deviation of the $\mathrm{BC}$ norm of 0.001823 indicate that the resultant mapping is actually of Teichmüller type. By the properties of Teichmüller map, the registration obtained is guaranteed to be bijective. This demonstrates that our proposed algorithm can effectively provide the unique registration result which minimizes the maximal conformality distortion. We have also computed the Teichmüller extremal mappings between a set of vertebral bones to construct the mean surface (Figure 9). Both feature points and the landmark curves are well-preserved, illustrating that the landmarks are consistently matched under the proposed registration algorithm.

To validate the invariance of the choice of cutting boundaries during the embedding process, we manually labeled two arbitrary simple closed loops (blue-red loops in Figure 10] with the same base point and run the proposed algorithm. Figure 11 shows the histogram of the optimal Teichmüller type BCs $\left|\mu_{g}\right|$ and $\left|\mu_{b r}\right|$ from the cases of green loops and blue-red loops respectively. Experiment shows that both registration results are coincident, with ||$\left|\mu_{\mathrm{g}}\right|-\left|\mu_{\mathrm{br}}\right| \|_{\infty}=0.0021$, indicating that our proposed algorithm is invariant to the initial choice of the cutting boundaries. We have also compared our implementation with rigid ICP and non-rigid ICP. The result is summarized in Table 1. For ease of comparison, we first normalize every vertebral bone to fit into a unit cube. In terms of the mean and maximum landmark matching errors $\left(e_{\text {mean }}, e_{\max }\right)$, our proposed method outperforms the two point-based registration methods. The Hausdorff distance $d_{H}$ between the registration result and the target also shows that our proposed method has a better overlay percentage to the target object. With the sacrifice of the registration accuracy, almost no conformality distortion is introduced by the rigid ICP, while the non-rigid ICP produces a large distortion of 0.9841 . Our proposed algorithm thus provides a balance between the computation requirement and the registration accuracy.
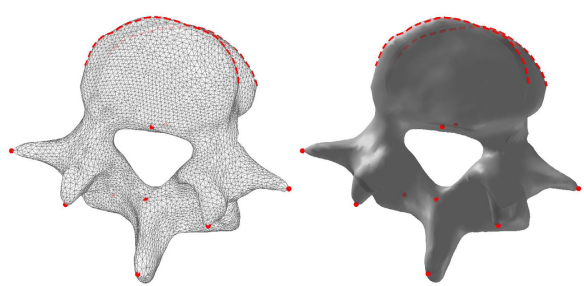

Fig. 9. Vertebral bone mean surface

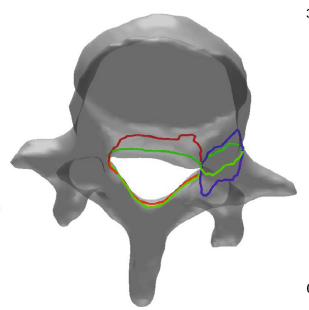

Fig. 10. Different loops

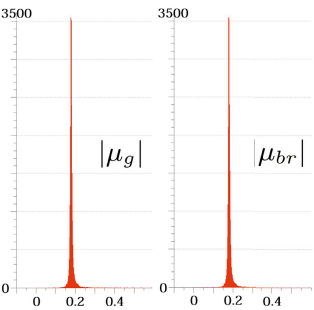

Fig. 11. $\left|\mu_{g}\right| \&\left|\mu_{b r}\right|$ 


\section{Conclusion and Future Works}

This paper presents a novel method to compute the Teichmüller extremal mapping with prescribed landmark correspondences between genus-1 surfaces, which minimizes the maximal conformality distortion. By the Teichmüller theory, existence and uniqueness of such mapping is guaranteed. We applied the proposed algorithm for the vertebral bone registration and the construction of mean surface of vertebral bones. Experimental results show that our method is effective in computing bijective feature aligned registration with smallest maximal conformality distortion. In the future, we plan to extend the proposed method to higher-genus surfaces and apply the method to more real applications in medical imaging for disease analysis.

Acknowledgements. This research is supported by the HKRGC GRF (CUHK Project ID: 404612).

\section{References}

1. Bookstein, F.L.: Principal Warps: Thin-Plate splines and the decomposition of deformations. IEEE Trans. Pattern Anal. Machine Intell. 11(6), 567-585 (1989)

2. Gardiner, F., Lakic, N.: Quasiconformal Teichmuller Theory. American Mathematics Society (2000)

3. Glaunès, J., Vaillant, M., Miller, M.I.: Landmark Matching via Large Deformation Diffeomorphisms on the Sphere. JMIV 20(1-2), 179-200 (2004)

4. Gu, X.F., Wang, Y., Chan, T.F., Thompson, P.M., Yau, S.T.: Genus zero surface conformal mapping and its application to brain surface mapping. IEEE Trans. Med. Imag. 23(8), 949-958 (2004)

5. Hurdal, M.K., Stephenson, K.: Discrete conformal methods for cortical brain flattening. Neuroimage 45(1), 86-98 (2009)

6. Jin, M., Kim, J., Luo, F., Gu, X.F.: Discrete surface Ricci flow. IEEE Trans. Visual. Comput. Graphics 14(5), 1030-1043 (2008)

7. Joshi, S., Miller, M.I.: Landmark matching via large deformation diffeomorphisms. IEEE Trans. Image Processing 9(8), 1357-1370 (2000)

8. Lui, L.M., Thiruvenkadam, S., Wang, Y., Chan, T.F., Thompson, P.M.: Optimized conformal parameterization of cortical surfaces using shape based matching of landmark curves. In: Metaxas, D., Axel, L., Fichtinger, G., Székely, G. (eds.) MICCAI 2008, Part I. LNCS, vol. 5241, pp. 494-501. Springer, Heidelberg (2008)

9. Lui, L.M., Wang, Y., Chan, T.F., Thompson, P.M.: Landmark constrained genus zero surface conformal mapping and its application to brain mapping research. Appl. Numer. Math. 57(5), 847-858 (2007)

10. Lui, L.M., Wen, C.F.: Geometric Registration of High-Genus Surfaces. SIIMS 7(1), 337-365 (2014)

11. Thompson, D.W.: On growth and form (1942)

12. Lui, L.M., Gu, X.F., Yau, S.T.: Convergence analysis of an iterative algorithm for Teichmüller maps via harmonic energy optimization. Math. Comp. (2014) 